\section{Targeting adenosine in SSc}

Aberrant activation of adenosinedependent signalling has been implicated in a number of fibrotic diseases, including systemic sclerosis (SSc). The results of a new study indicate that adenosine depletion with recombinant pegylated adenosine deaminase (PEG-ADA) improves the three cardinal features of SSc, namely fibrosis, inflammation and vasculopathy, in preclinical models of the disease.

PEG-ADA is currently used as enzyme replacement therapy for patients with adenosine deaminase severe combined immunodeficiency (ADA-SCID). "As PEG-ADA is already used for the treatment of ADA-SCID and is well tolerated even with long-term application, our findings could have clinical implications and stimulate clinical trials with PEG-ADA in SSc," notes Yun Zhang, first author of the study.

Targeting adenosine signalling in SSc has been attempted before, primarily by inactivation of $\mathrm{CD} 73$ (an ectonucleotidase important in adenosine production) or by targeting individual adenosine receptors. However, neither approach has yielded an effective treatment for SSc.

In the present study, Zhang and colleagues investigated the effects of PEG-ADA in two mouse models of SSc: Fra2-transgenic mice and the sclerodermatous chronic graft-versus-host disease (Scl-GVHD) mouse model. In both models, treatment with PEG-ADA inhibited myofibroblast differentiation and ameliorated fibrosis in the skin, lungs and intestines, organ systems commonly affected in SSc. "The antifibrotic effects in the mouse models were also translated to the human context using a full-thickness skin model," notes Zhang.

Treatment with PEG-ADA also prevented vascular manifestations in Fra2-transgenic mice and dampened

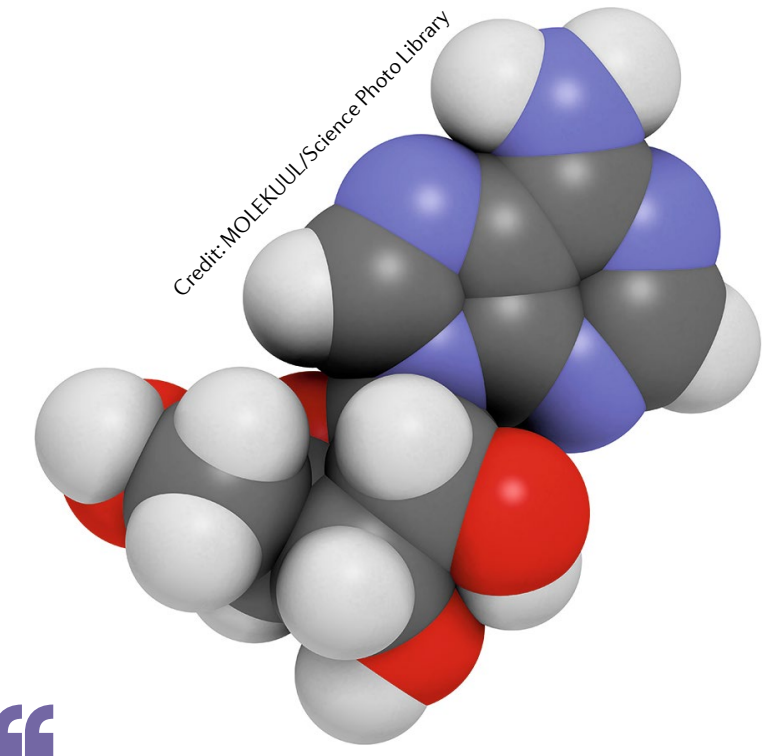

treatment

with PEG-ADA inhibited myofibroblast differentiation and ameliorated fibrosis in the skin, lungs and intestines 5

\title{
OSTEOARTHRITIS
}

\section{PDGF-BB is the key to unlocking pathological angiogenesis in OA}

Subchondral bone angiogenesis and neovascularization of the articular cartilage are features known to develop at an early stage during osteoarthritis (OA), but the exact mechanisms behind these processes have been unclear. A new study has uncovered a role for platelet-derived growth factor (PDGF)-BB in subchondral bone

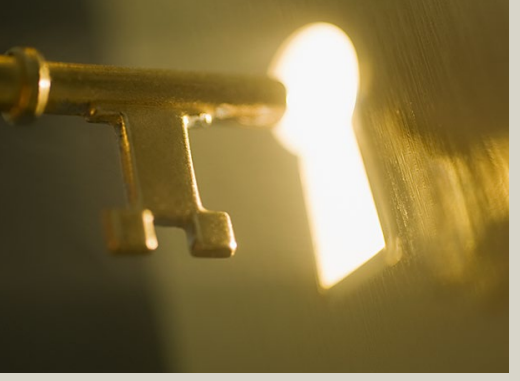

angiogenesis and in the development of OA in mice.

"Our study provides the first evidence that PDGF-BB derived from preosteoclasts is a key promoter of pathological subchondral bone angiogenesis during OA development," states corresponding author Mei Wan.

To assess the role of PDGF-BB in disease, Wan and colleagues used the destabilization of the medial meniscus (DMM) model of OA. "In mice after joint destabilization, mononuclear preosteoclasts in the subchondral bone secreted excessive amounts of PDGF-BB, which activated PDGF receptor- $\beta$ signalling in pericytes for neovessel formation," explains Wan.

The researchers used conditional PDGF-BB-knockout and transgenic mice that either lacked or overexpressed $P d g f b$ in osteoclast lineage cells. In this way, they could specifically assess the role of the inflammatory response in both the Scl-GVHD and Fra2-transgenic mouse models. RNA-Seq analysis demonstrated that PEG-ADA treatment normalized the expression of several genes related to fibrosis, vasculopathy and inflammation in Fra2-transgenic mice.

Sarah Onuora

ORIGINAL ARTICLE Zhang, Y. et al. Recombinant adenosine deaminase ameliorates inflammation, vascular disease and fibrosis in preclinical models of systemic sclerosis. Arthritis Rheumatol. https:// doi.org/10.1002/art.41259 (2020)

PDGF-BB production by preosteoclasts. "By using conditional PDGF-BB-knockout mice and conditional PDGF-BB transgenic mice, we found that PDGF-BB derived from preosteoclasts is both sufficient and necessary for the development of subchondral bone angiogenesis and subsequent joint degeneration," says Wan.

Conditional PDGF-BB-knockout

conditional PDGF-BB transgenic mice spontaneously developed OA-like disease

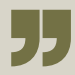

